\title{
Boundary Value Problem for the System Equations Mixed Type
}

\author{
Fayazov K.S., Khajiev I.O.* \\ Mathematics Faculty, National University of Uzbekistan, Universitet Str. 4, 100174 Tashkent, Uzbekistan
}

Copyright (C) 2016 by authors, all rights reserved. Authors agree that this article remains permanently

open access under the terms of the Creative Commons Attribution License 4.0 International License

\begin{abstract}
In this paper, we consider a system of equations of mixed type and with changing time direction. It is proved that solution of the system is not stable depend from the variation of the data. Theorems of uniqueness and conditional stability proved. The approximate solution constructed and numerical results are given.
\end{abstract}

Keywords System of Equations, Boundary Value Problem, Ill-posed Problem, a Priori Estimate, Theorem of the Uniqueness, Estimate of Conditional Stability, Approximate Solution, Regularization, Regularization's Parameter

\section{Introduction}

Consider the system of equations

$$
\left\{\begin{array}{l}
\left(\operatorname{sign} x \frac{\partial^{2}}{\partial t^{2}}+\frac{\partial^{2}}{\partial x^{2}}\right) v(x, t)=0 \\
\left(\operatorname{sign} x \frac{\partial}{\partial t}+\frac{\partial^{2}}{\partial x^{2}}\right) u(x, t)=v(x, t)
\end{array}\right.
$$

on the domain $\Omega=\{-1<x<1, x \neq 0,0<t<T\}$.

Problem. Find a solution $(u(x, t), v(x, t))$ of the system equations (1), satisfying the initial

$$
\left.\begin{array}{l}
u(x, 0)=f(x), \\
v(x, 0)=g(x), v_{t}(x, 0)=h(x)
\end{array}\right\},-1 \leq x \leq 1,
$$

the boundary conditions

$$
\left.\begin{array}{l}
u(-1, t)=u(1, t)=0 \\
v(-1, t)=v(1, t)=0
\end{array}\right\}, 0 \leq t \leq T
$$

and the gluing conditions

$$
\left.\begin{array}{l}
u(-0, t)=u(+0, t), \\
u_{x}(-0, t)=u_{x}(+0, t), \\
v(-0, t)=v(+0, t), \\
v_{x}(-0, t)=v_{x}(+0, t)
\end{array}\right\}, 0 \leq t \leq T .
$$

The investigate problem belongs to the class of ill-posed problems of mathematical physics. Correct problems for such equations considered by M. Gevrey, D. Pagani, G. Talenti, V. N. Vragov, V. K. Romanko, A.M. Nakhushev and others. See also works of N.V. Kislov, S. G. Pyatkov, A. I. Kozhanov, I.E. Egorov, A.A. Kerefov, I.S. Pulkin [1] and others.

The most important example of such type equations are mixed-type equation. A systematic study of them began with the work F.Tricomi and S. Gellerstedt. Shortly because of research S.A. Chaplygin and F.I. Frankl found out that mixed-type equations have important practical applications in the calculation of the flow of gas at around and supersonic speeds. Many important practical applications, such as jet aircraft and astronautics, rocketry, gas-dynamic lasers, caused an avalanche growth in boundary value problems of research for equations of mixed type, as a purely mathematical, and having applied nature.

Ill-posed problems investigated by H. Levine [2], A.L. Bukhgeim, K.S. Fayazov [5] and others.

In this paper we study the conditional correctness, prove the theorem on conditional stability and uniqueness of the solution of problem (1) - (4) and construct an approximate solution by the regularization method. We estimate norm of the difference between exact and regularized solutions.

Definition. By solution of the problem (1)-(4) we understand a pair of functions $(u, v)$ having respective continuous derivatives involved in the system of equations and satisfy the system of equations (1) and the conditions (2) - (4).

\section{The main results}

For further discussion, we need in the following lemmas: Lemma 1[6]. Let $v(x, t)$ satisfies the equation

$$
\operatorname{sign} x v_{t t}(x, t)+v_{x x}(x, t)=0
$$

and conditions $v(-1, t)=v(1, t)=0, v(-0, t)=$ $v(+0, t), v_{x}(-0, t)=v_{x}(+0, t)$, then for $v(x, t)$ for every $0<t<T$ the following estimate

$\|v(x, t)\|^{2} \leq 2\left(\|v(x, 0)\|^{2}+p\right)^{\frac{T-t}{T}}\left(\|v(x, T)\|^{2}+p\right)^{\frac{t}{T}} e^{2 t(T-t)}$,

is true, where $p=\frac{1}{2}\left(\left\|v_{x x}(x, 0)\right\|^{2}+\left\|v_{t}(x, 0)\right\|^{2}\right)$.

Definition of norm and Proof of Lemma 1 one can find in [6]. 
Lemma 2. Let $u(x, t)$ satisfies the equation

$$
\operatorname{sign} x u_{t}(x, t)+u_{x x}(x, t)=v(x, t)
$$

and conditions $u(-1, t)=u(1, t)=0, u(-0, t)=$ $u(+0, t), u_{x}(-0, t)=u_{x}(+0, t)$, then for $u(x, t)$ for every $0<t<T$ the following estimate

$$
\begin{gathered}
\|u(x, t)\|^{2} \leq 2\left(\|u(x, 0)\|^{2}+\int_{0}^{T}\|v(x, t)\|^{2} d t\right)^{\frac{T-t}{T}} \times \\
\left(\|u(x, T)\|^{2}+\int_{0}^{T}\|v(x, t)\|^{2} d t\right)^{\frac{t}{T}}+\int_{0}^{T}\|v(x, t)\|^{2} d t,
\end{gathered}
$$

is valid.

Proof. Solution of the equation (5) one can present as the sum

$$
u(x, t)=\bar{u}(x, t)+\tilde{u}(x, t),
$$

where $\bar{u}(x, t)$ solution of the homogeneous equation

$$
\operatorname{signx} \bar{u}_{t}(x, t)+\bar{u}_{x x}(x, t)=0,
$$

$\tilde{u}(x, t)$ - a particular solution of the inhomogeneous equation

$$
\operatorname{signx} \tilde{u}_{t}(x, t)+\tilde{u}_{x x}(x, t)=v(x, t) .
$$

Moreover functions $\bar{u}(x, t), \tilde{u}(x, t)$ satisfy the boundary conditions

$$
\bar{u}(-1, t)=\bar{u}(1, t)=0, \quad \tilde{u}(-1, t)=\tilde{u}(1, t)=0,
$$

and gluing conditions

$$
\begin{aligned}
& \bar{u}(-0, t)=\bar{u}(+0, t), \quad \bar{u}_{x}(-0, t)=\bar{u}_{x}(+0, t), \\
& \tilde{u}(-0, t)=\tilde{u}(+0, t), \tilde{u}_{x}(-0, t)=\tilde{u}_{x}(+0, t) .
\end{aligned}
$$

According to the results of $[5,6]$ solutions $\bar{u}(x, t)$ and $\tilde{u}(x, t)$ can be presented in the form

$$
\begin{aligned}
& \bar{u}(x, t)=\sum_{k=1}^{\infty} \bar{u}_{k}^{+}(t) X_{k}^{+}(x)+\sum_{k=1}^{\infty} \bar{u}_{k}^{-}(t) X_{k}^{-}(x), \\
& \tilde{u}(x, t)=\sum_{k=1}^{\infty} \tilde{u}_{k}^{+}(t) X_{k}^{+}(x)+\sum_{k=1}^{\infty} \tilde{u}_{k}^{-}(t) X_{k}^{-}(x),
\end{aligned}
$$

here $\bar{u}_{k}^{ \pm}(t), \tilde{u}_{k}^{ \pm}(t)$ for each $k=1,2, \ldots$ satisfy the following problems, respectively:

$$
\begin{aligned}
& \left\{\begin{array}{l}
\left\{\tilde{u}_{k}^{+}(t)\right\}_{t}-\lambda_{k}^{+} \tilde{u}_{k}^{+}(t)=v_{k}^{+}(t), \\
\tilde{u}_{k}^{+}(0)=-\int_{0}^{T} e^{-\lambda_{k}^{+} \tau} v_{k}^{+}(\tau) d \tau,
\end{array}\right. \\
& \left\{\begin{array}{l}
\left\{\tilde{u}_{k}^{-}(t)\right\}_{t}-\lambda_{k}^{-} \tilde{u}_{k}^{-}(t)=v_{k}^{-}(t), \\
\tilde{u}_{k}^{-}(0)=0,
\end{array}\right.
\end{aligned}
$$

and

$$
\begin{gathered}
\left\{\begin{array}{l}
\left\{\bar{u}_{k}^{+}(t)\right\}_{t}-\lambda_{k}^{+} \bar{u}_{k}^{+}(t)=0, \\
\bar{u}_{k}^{+}(0)=f_{k}^{+}+\int_{0}^{T} e^{-\lambda_{k}^{+} \tau} v_{k}^{+}(\tau) d \tau,
\end{array}\right. \\
\left\{\begin{array}{l}
\left\{\bar{u}_{k}^{-}(t)\right\}_{t}-\lambda_{k}^{-} \bar{u}_{k}^{-}(t)=0, \\
\bar{u}_{k}^{-}(0)=f_{k}^{-},
\end{array}\right.
\end{gathered}
$$

where $v_{k}^{ \pm}(t)=\int_{-1}^{1} \operatorname{signx} v(x, t) X_{k}^{ \pm}(x) d x, f_{k}^{ \pm}=$ $\int_{-1}^{1} \operatorname{signx} f(x) X_{k}^{ \pm}(x) d x, X_{k}^{ \pm}(x)$ - eigenfunctions, corresponding respectively to positive $\lambda_{k}^{+}$and negative $\lambda_{k}^{-}$eigenvalues, and numbers $\lambda_{k}^{+},-\lambda_{k}^{-}$constitute non-decreasing sequence. Notice, that $X_{k}^{ \pm}(x)$ and $\lambda_{k}^{ \pm}$eigenfunctions and eigenvalues of the following problem:

$$
\left\{\begin{array}{l}
\text { signx } X^{\prime \prime}(x)+\lambda X(x)=0, \\
X(-1)=0, \quad X(1)=0, \\
X(-0)=X(+0), \\
X^{\prime}(-0)=X^{\prime}(+0) .
\end{array}\right.
$$

It is easy to note [3], that

$$
\begin{gathered}
\tilde{u}_{k}^{+}(t)=-\int_{t}^{T} e^{\lambda_{k}^{+}(t-\tau)} v_{k}^{+}(\tau) d \tau, \\
\tilde{u}_{k}^{-}(t)=\int_{0}^{t} e^{\lambda_{k}^{-}(t-\tau)} v_{k}^{-}(\tau) d \tau,
\end{gathered}
$$

from here you can get

$$
\begin{array}{r}
\|\tilde{u}(x, t)\|^{2}=\sum_{k=1}^{\infty}\left\{\tilde{u}_{k}^{+}(t)\right\}^{2}+\left\{\tilde{u}_{k}^{-}(t)\right\}^{2} \leq \\
\int_{0}^{T}\|v(x, t)\|^{2} d t .
\end{array}
$$

Next, using the logarithmic convexity of the functions $\bar{u}_{k}^{ \pm}(t)$ for each $k=1,2, \ldots$, we have the following estimates

$$
\begin{aligned}
& \left\{\bar{u}_{k}^{+}(t)\right\}^{2} \leq\left(\left\{\bar{u}_{k}^{+}(0)\right\}^{2}\right)^{\frac{T-t}{T}}\left(\left\{\bar{u}_{k}^{+}(T)\right\}^{2}\right)^{\frac{t}{T}}, \\
& \left\{\bar{u}_{k}^{-}(t)\right\}^{2} \leq\left(\left\{\bar{u}_{k}^{-}(0)\right\}^{2}\right)^{\frac{T-t}{T}}\left(\left\{\bar{u}_{k}^{-}(T)\right\}^{2}\right)^{\frac{t}{T}} .
\end{aligned}
$$

Summing over $k, k=1,2, \ldots$ and using the Holder inequality for the sum we have

$$
\|\bar{u}(x, t)\|^{2} \leq 2\left(\|\bar{u}(x, 0)\|^{2}\right)^{\frac{T-t}{T}}\left(\|\bar{u}(x, T)\|^{2}\right)^{\frac{t}{T}}
$$

and that $\bar{u}(x, t)=u(x, t)-\tilde{u}(x, t)$, then

$$
\begin{aligned}
\|u(x, t)\|^{2} & \leq 2\left(\|u(x, 0)\|^{2}+\|\tilde{u}(x, 0)\|^{2}\right)^{\frac{T-t}{T}} \times \\
& \left(\|u(x, T)\|^{2}+\|\tilde{u}(x, T)\|^{2}\right)^{\frac{t}{T}}+\|\tilde{u}(x, t)\|^{2},
\end{aligned}
$$

taking into account (6) from this we obtain required inequality. The Lemma 2 proved.

Set of correctness $\mathrm{M}$ is defined as follows

$$
M=\left\{(u, v):\|u(x, T)\|^{2}+\|v(x, T)\|^{2} \leq m^{2}\right\} .
$$

Theorem 1. Let the solution of the problem (1) - (4) exists and $(u(x, t), v(x, t)) \in M$, then the solution of the problem is unique.

Proof. Let two pair of functions $\left(u_{1}(x, t), v_{1}(x, t)\right)$, $\left(u_{2}(x, t), v_{2}(x, t)\right)$ are solutions of problem (1) - (4). We denote $u(x, t)=u_{1}(x, t)-u_{2}(x, t), v(x, t)=v_{1}(x, t)-$ $v_{2}(x, t)$. Then pair of functions $(u(x, t), v(x, t))$ satisfies the system of equations (1) and the homogeneous conditions (2) - (4). From the result of Lemma 1 it is easy to see $\|v(x, t)\|=0$ then $v(x, t)=0$, and on the base of Lemma 2 , we get $\|u(x, t)\|=0$, from here $u(x, t)=0$ for any $(x, t) \in \Omega$. Then $v_{1}(x, t) \equiv v_{2}(x, t), u_{1}(x, t) \equiv u_{2}(x, t)$, solution of problem (1) - (4) is unique. The theorem proved.

Theorem 2. Let the solution of the problem (1) (4) exist, $(u(x, t), v(x, t)) \in M,\left\|f(x)-f_{\varepsilon}(x)\right\| \leq \varepsilon$, 
$\left\|g(x)-g_{\varepsilon}(x)\right\|_{W_{2}^{2}} \leq \varepsilon,\left\|h(x)-h_{\varepsilon}(x)\right\| \leq \varepsilon$. Then the solution of problem (1) - (4) for every $t \in(0, T)$ satisfies the inequalities

$$
\|v(x, t)\|^{2} \leq 2\left(2 \varepsilon^{2}\right)^{\frac{T-t}{T}}\left(m^{2}+\varepsilon^{2}\right)^{\frac{t}{T}} e^{2 t(T-t)},
$$

$\|u(x, t)\|^{2} \leq 2\left(\varepsilon^{2}+\gamma(m, \varepsilon)\right)^{\frac{T-t}{T}}\left(m^{2}+\gamma(m, \varepsilon)\right)^{\frac{t}{T}}+\gamma(m, \varepsilon)$,

where $\gamma(m, \varepsilon)=2 \int_{0}^{T}\left(2 \varepsilon^{2}\right)^{\frac{T-t}{T}}\left(m^{2}+\varepsilon^{2}\right)^{\frac{t}{T}} e^{2 t(T-t)} d t$.

Proof. On the base of the conditions of Theorem pair of functions $(u(x, t), v(x, t))$ satisfies the system of equations

$$
\left\{\begin{array}{l}
\left(\operatorname{sign} x \frac{\partial^{2}}{\partial t^{2}}+\frac{\partial^{2}}{\partial x^{2}}\right) v(x, t)=0 \\
\left.\operatorname{sign} x \frac{\partial}{\partial t}+\frac{\partial^{2}}{\partial x^{2}}\right) u(x, t)=v(x, t)
\end{array}\right.
$$

on domain $\Omega=\{-1<x<1, x \neq 0,0<t<T\}$ and conditions: initial

$$
\left.\begin{array}{l}
u(x, 0)=f(x)-f_{\varepsilon}(x), \\
v(x, 0)=g(x)-g_{\varepsilon}(x), \\
v_{t}(x, 0)=h(x)-h_{\varepsilon}(x)
\end{array}\right\},-1 \leq x \leq 1,
$$

boundary

$$
\left.\begin{array}{l}
u(-1, t)=u(1, t)=0 \\
v(-1, t)=v(1, t)=0
\end{array}\right\}, 0 \leq t \leq T
$$

and gluing conditions

$$
\left.\begin{array}{l}
u(-0, t)=u(+0, t), \\
u_{x}(-0, t)=u_{x}(+0, t), \\
v(-0, t)=v(+0, t), \\
v_{x}(-0, t)=v_{x}(+0, t)
\end{array}\right\}, 0 \leq t \leq T
$$

From the initial conditions, we obtain the following

$$
\begin{gathered}
\|v(x, 0)\|=\left\|g(x)-g_{\varepsilon}(x)\right\| \leq \varepsilon, \\
\|u(x, 0)\|=\left\|f(x)-f_{\varepsilon}(x)\right\| \leq \varepsilon, \\
p=\frac{1}{2}\left(\left\|v_{x x}(x, 0)\right\|^{2}+\left\|v_{t}(x, 0)\right\|^{2}\right)= \\
\frac{1}{2}\left(\left\|g^{\prime \prime}(x)-g^{\prime \prime}{ }_{\varepsilon}(x)\right\|^{2}+\left\|h(x)-h_{\varepsilon}(x)\right\|^{2}\right) \leq \varepsilon^{2} .
\end{gathered}
$$

Since from (7)

$$
\|v(x, T)\| \leq m,\|u(x, T)\| \leq m,
$$

then, according to Lemma 1

$$
\|v(x, t)\|^{2} \leq 2\left(2 \varepsilon^{2}\right)^{\frac{T-t}{T}}\left(m^{2}+\varepsilon^{2}\right)^{\frac{t}{T}} e^{2 t(T-t)},
$$

and from Lemma 2

$\|u(x, t)\|^{2} \leq 2\left(\varepsilon^{2}+\gamma(m, \varepsilon)\right)^{\frac{T-t}{T}}\left(m^{2}+\gamma(m, \varepsilon)\right)^{\frac{t}{T}}+\gamma(m, \varepsilon)$,

where $\gamma(m, \varepsilon)=2 \int_{0}^{T}\left(2 \varepsilon^{2}\right)^{\frac{T-t}{T}}\left(m^{2}+\varepsilon^{2}\right)^{\frac{t}{T}} e^{2 t(T-t)} d t$.

Remark. The results of this work can easily be extended to equation that is more general.

\section{Approximate solution}

The approximate solution for accurate data defined as follows

$$
\begin{gathered}
u^{N}(x, t)=\sum_{k=1}^{N}\left(f_{k}^{+} e^{\lambda_{k}^{+} t}+\int_{0}^{T} e^{\lambda_{k}^{+}(t-\tau)} v_{k}^{+}(\tau) d \tau-\right. \\
\left.\int_{t}^{T} e^{\lambda_{k}^{+}(t-\tau)} v_{k}^{+}(\tau) d \tau\right) X_{k}^{+}(x)+ \\
+\sum_{k=1}^{N}\left(f_{k}^{-} e^{\lambda_{k}^{-} t}+\int_{0}^{t} e^{\lambda_{k}^{-}(t-\tau)} v_{k}^{-}(\tau) d \tau\right) X_{k}^{-}(x), \\
v^{N}(x, t)=\sum_{k=1}^{N}\left(g_{k}^{+} \cosh \sqrt{\lambda_{k}^{+}} t+\frac{h_{k}^{+}}{\sqrt{\lambda_{k}^{+}}} \sinh \sqrt{\lambda_{k}^{+} t}\right) X_{k}^{+}+ \\
\sum_{k=1}^{N}\left(g_{k}^{-} \cos \sqrt{\lambda_{k}^{-}} t+\frac{h_{k}^{-}}{\sqrt{-\lambda_{k}^{-}}} \sin \sqrt{-\lambda_{k}^{-}} t\right) X_{k}^{-}
\end{gathered}
$$

where

$$
\begin{gathered}
f_{k}^{ \pm}=\int_{-1}^{1} \operatorname{signx} f(x) X_{k}^{ \pm}(x) d x, \\
g_{k}^{ \pm}=\int_{-1}^{1} \operatorname{signx} g(x) X_{k}^{ \pm}(x) d x, \\
h_{k}^{ \pm}=\int_{-1}^{1} \operatorname{signx} h(x) X_{k}^{ \pm}(x) d x, \\
v_{k}^{ \pm}(t)=\int_{-1}^{1} \operatorname{signx} v(x, t) X_{k}^{ \pm}(x) d x,
\end{gathered}
$$

$N$ - integer parameter regularization.

The approximate solution of the approximate data defined as follows

$$
\begin{gathered}
u_{\varepsilon}^{N}(x, t)=\sum_{k=1}^{N}\left(f_{k_{\varepsilon}}^{+} e^{\lambda_{k}^{+} t}+\int_{0}^{T} e^{\lambda_{k}^{+}(t-\tau)} v_{k_{\varepsilon}}^{+}(\tau) d \tau-\right. \\
\left.\int_{t}^{T} e^{\lambda_{k}^{+}(t-\tau)} v_{k_{\varepsilon}}^{+}(\tau) d \tau\right) X_{k}^{+}(x)+ \\
+\sum_{k=1}^{N}\left(f_{k_{\varepsilon}}^{-} e^{\lambda_{k}^{-} t}+\int_{0}^{t} e^{\lambda_{k}^{-}(t-\tau)} v_{k_{\varepsilon}}^{-}(\tau) d \tau\right) X_{k}^{-}(x),
\end{gathered}
$$

$$
\begin{aligned}
& v_{\varepsilon}^{N}(x, t)=\sum_{k=1}^{N}\left(g_{k_{\varepsilon}}^{+} \cosh \sqrt{\lambda_{k}^{+}} t+\frac{h_{k_{\varepsilon}}^{+}}{\sqrt{\lambda_{k}^{+}}} \sinh \sqrt{\lambda_{k}^{+}} t\right) X_{k}^{+}+ \\
& \sum_{k=1}^{N}\left(g_{k_{\varepsilon}}^{-} \cos \sqrt{\lambda_{k}^{-}} t+\frac{h_{k_{\varepsilon}}^{-}}{\sqrt{-\lambda_{k}^{-}}} \sin \sqrt{-\lambda_{k}^{-}} t\right) X_{k}^{-}
\end{aligned}
$$

where

$$
\begin{aligned}
& f_{k_{\varepsilon}}^{ \pm}=\int_{-1}^{1} \operatorname{signx} f_{\varepsilon}(x) X_{k}^{ \pm}(x) d x, \\
& g_{k_{\varepsilon}}^{ \pm}=\int_{-1}^{1} \operatorname{signx} g_{\varepsilon}(x) X_{k_{\varepsilon}}^{ \pm}(x) d x, \\
& h_{k_{\varepsilon}}^{ \pm}=\int_{-1}^{1} \operatorname{signx} h_{\varepsilon}(x) X_{k}^{ \pm}(x) d x,
\end{aligned}
$$




$$
v_{k_{\varepsilon}}^{ \pm}(t)=\int_{-1}^{1} \operatorname{signx} v_{\varepsilon}(x, t) X_{k}^{ \pm}(x) d x .
$$

Let $\left\|f(x)-f_{\varepsilon}(x)\right\| \leq \varepsilon,\left\|g(x)-g_{\varepsilon}(x)\right\| \leq \varepsilon$, $\left\|h(x)-h_{\varepsilon}(x)\right\| \leq \varepsilon(u(x, t), v(x, t)) \in M$. Then we estimate the norm of the difference between of exact and approximate solutions by the way

$$
\begin{gathered}
\left\|u(x, t)-u_{\varepsilon}^{N}(x, t)\right\| \leq\left\|u(x, t)-u^{N}(x, t)\right\|+ \\
\left\|u^{N}(x, t)-u_{\varepsilon}^{N}(x, t)\right\|, \\
\left\|v(x, t)-v_{\varepsilon}^{N}(x, t)\right\| \leq\left\|v(x, t)-v^{N}(x, t)\right\|+ \\
\left\|v^{N}(x, t)-v_{\varepsilon}^{N}(x, t)\right\| .
\end{gathered}
$$

1. Let $g(x)=0$. We estimate the second term on the right side of inequality (13) by way

$$
\begin{gathered}
\left\|v^{N}(x, t)-v_{\varepsilon}^{N}(x, t)\right\|^{2} \leq \sum_{k=1}^{N} \frac{1}{\lambda_{k}^{+}}\left(h_{k}^{+}-h_{k_{\varepsilon}}^{+}\right)^{2} \sinh ^{2} \sqrt{\lambda_{k}^{+}} t \\
+\frac{1}{-\lambda_{k}^{-}}\left(h_{k}^{-}-h_{k_{\varepsilon}}^{-}\right)^{2} \sin ^{2} \sqrt{-\lambda_{k}^{-}} t \leq \frac{\sinh ^{2} \sqrt{\lambda_{N}^{+}} t}{\lambda_{1}^{+}} \varepsilon^{2} .
\end{gathered}
$$

It is not difficult to see

$$
\begin{gathered}
\left\|v(x, t)-v^{N}(x, t)\right\|^{2} \leq \sum_{k=N+1}^{\infty} \frac{1}{\lambda_{k}^{+}}\left\{h_{k}^{+}\right\}^{2} \sinh ^{2} \sqrt{\lambda_{k}^{+}} t+ \\
\sum_{k=N+1}^{\infty} \frac{1}{-\lambda_{k}^{-}}\left\{h_{k}^{-}\right\}^{2} \sin ^{2} \sqrt{-\lambda_{k}^{-}} t .
\end{gathered}
$$

From (7) follow $\|v(x, T)\| \leq m$, then easy to see that

$$
\sum_{k=1}^{\infty} \frac{1}{\lambda_{k}^{+}}\left\{h_{k}^{+}\right\}^{2} \sinh ^{2} \sqrt{\lambda_{k}^{+}} T \leq m^{2} .
$$

Right sides the array in (14) reaches his maximum under the condition (15), if

$$
h_{k}^{+}=\left\{\begin{array}{l}
0, k \neq N+1, \\
\frac{m \sqrt{\lambda_{k}^{+}}}{\sinh \sqrt{\lambda_{k}^{+}} T}, k=N+1
\end{array},\right.
$$

then

$$
\left\|v(x, t)-v^{N}(x, t)\right\|^{2} \leq \frac{m^{2} \sinh ^{2} \sqrt{\lambda_{N+1}^{+}} t}{\sinh ^{2} \sqrt{\lambda_{N+1}^{+} T}}+\alpha(N),
$$

where $\alpha(N)=\sum_{k=N+1}^{\infty}\left\{h_{k}^{-}\right\}^{2}$, that $\alpha(N) \rightarrow 0$, by $N \rightarrow \infty$, since the corresponding series for $h(x)$ is convergent.

We now estimate the right side of inequality (12). Consider the second term

$$
\begin{gathered}
\left\|u^{N}(x, t)-u_{\varepsilon}^{N}(x, t)\right\|^{2}= \\
\sum_{k=1}^{N}\left(\int_{0}^{t} e^{\lambda_{k}^{+}(t-\tau)}\left(v_{k}^{+}(\tau)-v_{k_{\varepsilon}}^{+}(\tau)\right) d \tau\right)^{2}+ \\
\sum_{k=1}^{N}\left(\int_{0}^{t} e^{\lambda_{k}^{-}(t-\tau)}\left(v_{k}^{-}(\tau)-v_{k_{\varepsilon}}^{-}(\tau)\right) d \tau\right)^{2} \leq \\
\frac{e^{2 \lambda_{N}^{+} t}}{2 \lambda_{1}^{+}} \int_{0}^{t}\left\|v^{N}(x, t)-v_{\varepsilon}^{N}(x, t)\right\|^{2} d \tau \leq \\
\frac{e^{2 \lambda_{N}^{+} t}}{4\left\{\lambda_{1}^{+}\right\}^{2}} \int_{0}^{t} \sinh ^{2} \sqrt{\lambda_{N}^{+}} \tau d \tau \varepsilon^{2} .
\end{gathered}
$$

Now, from the definition of the norm

$$
\begin{gathered}
\left\|u(x, t)-u^{N}(x, t)\right\|^{2}= \\
\sum_{k=N+1}^{\infty}\left(\left(f_{k}^{+}+\int_{0}^{T} e^{-\lambda_{k}^{+} \tau} v_{k}^{+}(\tau) d \tau\right) e^{\lambda_{k}^{+} t}-\right. \\
\left.\quad \int_{t}^{T} e^{\lambda_{k}^{+}(t-\tau)} v_{k}^{+}(\tau) d \tau\right)^{2}+ \\
\sum_{k=N+1}^{\infty}\left(f_{k}^{-} e^{\lambda_{k}^{-} t}+\int_{0}^{t} e^{\lambda_{k}^{-}(t-\tau)} v_{k}^{-}(\tau) d \tau\right)^{2} .
\end{gathered}
$$

Notice, that $\|u(x, T)\| \leq m$ or

$$
\sum_{k=1}^{\infty}\left(f_{k}^{+}+\int_{0}^{T} e^{-\lambda_{k}^{+} \tau} v_{k}^{+}(\tau) d \tau\right)^{2} e^{2 \lambda_{k}^{+} T} \leq m^{2} .
$$

We estimate (17) under the condition (18). It is easy to notice that its maximum value reaches under the condition

$$
\left\{\begin{array}{l}
f_{N+1}^{+}=m e^{-\lambda_{N+1}^{+} T}-\int_{0}^{T} e^{-\lambda_{N+1}^{+} \tau} v_{N+1}^{+}(\tau) d \tau \\
f_{k}^{+}=-\int_{0}^{T} e^{-\lambda_{k}^{+} \tau} v_{k}^{+}(\tau) d \tau, k \neq N+1
\end{array}\right.
$$

Using this fact we estimate (17) under (16)

$$
\begin{aligned}
\left\|u(x, t)-u^{N}(x, t)\right\|^{2} \leq 2 m^{2} e^{\lambda_{N+1}^{+}(t-T)}+ \\
2 m^{2} \int_{0}^{T} \frac{\sinh ^{2} \sqrt{\lambda_{N+1}^{+}} \tau}{\sinh ^{2} \sqrt{\lambda_{N+1}^{+}} T} d \tau+\alpha(N) T+\beta(N)
\end{aligned}
$$

where $\beta(N)=\sum_{N+1}^{\infty}\left\{f_{k}^{-}\right\}^{2}$. Notice $\beta(N) \rightarrow 0$ at $N \rightarrow \infty$. At the final we get estimate

$$
\begin{aligned}
& \left\|u(x, t)-u_{\varepsilon}^{N}(x, t)\right\|^{2} \leq 2 m^{2} e^{\lambda_{N+1}^{+}(t-T)}+ \\
& \frac{m^{2}}{\sqrt{\lambda_{N+1}^{+}}} \operatorname{coth} \sqrt{\lambda_{N+1}^{+}} T+\alpha(N) T+ \\
& \frac{e^{2 \lambda_{N}^{+} t} \sinh 2 \sqrt{\lambda_{N}^{+}} t}{8\left\{\lambda_{1}^{+}\right\}^{2} \sqrt{\lambda_{N}^{+}}} \varepsilon^{2}+\beta(N) \\
& \left\|v(x, t)-v_{\varepsilon}^{N}(x, t)\right\|^{2} \leq \frac{m^{2} \sinh ^{2} \sqrt{\lambda_{N+1}^{+}} t}{\sinh ^{2} \sqrt{\lambda_{N+1}^{+} T}}+ \\
& \frac{\sinh ^{2} \sqrt{\lambda_{N}^{+}} t}{\lambda_{1}^{+}} \varepsilon^{2}+\alpha(N)
\end{aligned}
$$

2. Now consider the case $h(x)=0$. We have

$$
\begin{gathered}
\left\|v^{N}(x, t)-v_{\varepsilon}^{N}(x, t)\right\|^{2} \leq \sum_{k=1}^{N}\left(g_{k}^{+}-g_{k_{\varepsilon}}^{+}\right)^{2} \cos ^{2} \sqrt{\lambda_{k}^{+}} t+ \\
\sum_{k=1}^{N}\left(g_{k}^{-}-g_{k_{\varepsilon}}^{-}\right)^{2} \cos ^{2} \sqrt{-\lambda_{k}^{-}} t \leq \cosh ^{2} \sqrt{\lambda_{N}^{+}} t \varepsilon^{2} .
\end{gathered}
$$


Using conditions $\|v(x, T)\| \leq m$ similarly as the above we obtain

$$
\left\|v(x, t)-v^{N}(x, t)\right\|^{2} \leq \frac{m^{2} \cosh ^{2} \sqrt{\lambda_{N+1}^{+}} t}{\cosh ^{2} \sqrt{\lambda_{N+1}^{+}} T}+\gamma(N)
$$

where $\gamma(N)=\sum_{k=N+1}^{\infty}\left\{g_{k}^{-}\right\}^{2}$. Now we get estimate for expression of (12) under the condition $(u(x, t), v(x, t)) \in M$. Acting by similar way as above we have the following estimates

$$
\begin{aligned}
& \left\|u^{N}(x, t)-u_{\varepsilon}^{N}(x, t)\right\|^{2} \leq \\
& \frac{e^{2 \lambda_{N}^{+} t}}{2 \lambda_{1}^{+}} \int_{0}^{t}\left\|v^{N}(x, t)-v_{\varepsilon}^{N}(x, t)\right\|^{2} d \tau \leq \\
& \quad \frac{e^{2 \lambda_{N}^{+} t}}{2 \lambda_{1}^{+}} \int_{0}^{t} \cosh ^{2} \sqrt{\lambda_{N}^{+}} \tau d \tau \varepsilon^{2}, \\
& \left\|u(x, t)-u^{N}(x, t)\right\|^{2} \leq 2 m^{2} e^{\lambda_{N+1}^{+}(t-T)}+ \\
& 2 m^{2} \int_{0}^{T} \frac{\cosh ^{2} \sqrt{\lambda_{N+1}^{+}} t}{\cosh ^{2} \sqrt{\lambda_{N+1}^{+}} T} d \tau+\gamma(N) T+\beta(N) .
\end{aligned}
$$

As result the estimate of the norm difference between of exact and approximate solutions will be given in this form

$$
\begin{aligned}
& \left\|u(x, t)-u_{\varepsilon}^{N}(x, t)\right\|^{2} \leq 2 m^{2} e^{\lambda_{N+1}^{+}(t-T)}+ \\
& \frac{m^{2}}{\sqrt{\lambda_{N+1}^{+}}} \operatorname{coth} \sqrt{\lambda_{N+1}^{+}} T+\gamma(N) T+ \\
& \frac{e^{2 \lambda_{N}^{+} t}}{2 \lambda_{1}^{+}}\left(\frac{1}{2 \sqrt{\lambda_{N}^{+}}} \sinh 2 \sqrt{\lambda_{N}^{+}} t+t\right) \varepsilon^{2}+\beta(N), \\
& \left\|v(x, t)-v_{\varepsilon}^{N}(x, t)\right\|^{2} \leq \frac{2 m^{2} \cosh ^{2} \sqrt{\lambda_{N+1}^{+}} t}{\cosh ^{2} \sqrt{\lambda_{N+1}^{+}} T}+ \\
& \cosh ^{2} \sqrt{\lambda_{N}^{+}} t \varepsilon^{2}+\gamma(N) .
\end{aligned}
$$

Minimizing right side we obtain formula for the regularization parameter, which we will use for numerical calculation.

\section{Numerical calculations}

For the numerical solution of the problem (1) - (4) we take the initial data in the form

$$
f(x)=x^{2}-1, g(x)=0, h(x)=\sin \pi x, T=0,25,
$$

as well as the approximate data can be taken in the form

$f(x)=\left(x^{2}-1\right)(1+\varepsilon), h(x)=\sin \pi x(1+\varepsilon), \varepsilon=0,0001$.

The below graphs and tables of calculations show that the values of approximate solutions with exact data and the values of approximate solutions with approximate data are nearly close to each other.
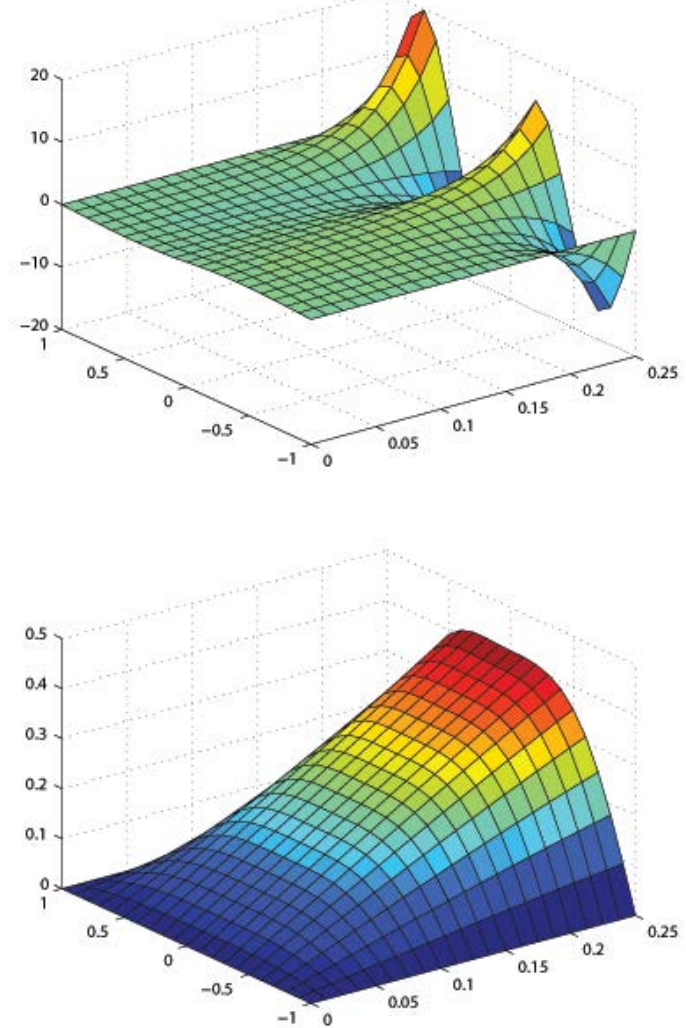

Figure 1. The Graph of approximate solution $\left(u^{N}(x, t), v^{N}(x, t)\right)$ for accurate data.
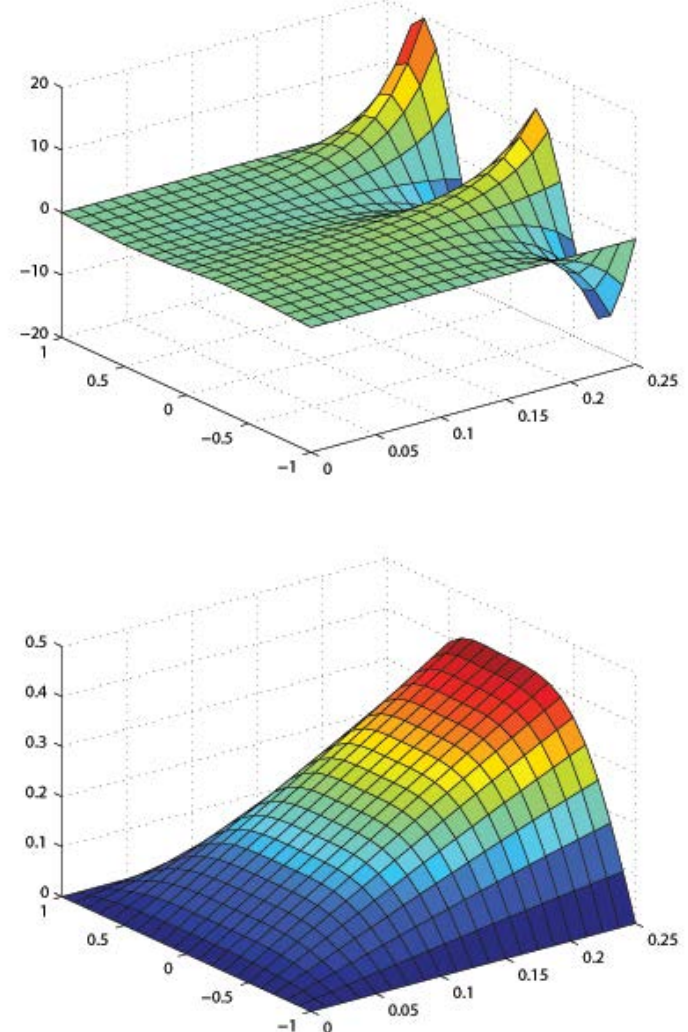

Figure 2. The Graph of approximate solution $\left(u_{\varepsilon}^{N}(x, t), v_{\varepsilon}^{N}(x, t)\right)$ for approximate data 
Table 1. Approximate solution $\left(u^{N}(x, t), v^{N}(x, t)\right)$ for accurate data

\begin{tabular}{c|c|c|c|c}
\hline & $\mathrm{t}=0,05$ & $\mathrm{t}=0,1$ & $\mathrm{t}=0,2$ & $\mathrm{t}=0,25$ \\
\hline $\mathrm{x}=-1$ & 0 & 0 & 0 & 0 \\
\hline $\mathrm{x}=-0,7$ & 0,51274 & 0,60606 & $-2,29596$ & $-15,399$ \\
\hline $\mathrm{x}=-0,4$ & 0,74791 & 1,07252 & 2,21422 & 3,73774 \\
\hline $\mathrm{x}=0$ & 0,08614 & 0,16516 & $-0,14539$ & $-1,77194$ \\
\hline $\mathrm{x}=0,4$ & $-0,57518$ & $-0,69912$ & $-1,4733$ & $-3,22008$ \\
\hline $\mathrm{x}=0,7$ & $-0,38694$ & $-0,29293$ & 3,84206 & 19,74447 \\
\hline $\mathrm{x}=1$ & 0 & 0 & 0 & 0 \\
\hline \hline & $\mathrm{t}=0,05$ & $\mathrm{t}=0,1$ & $\mathrm{t}=0,2$ & $\mathrm{t}=0,25$ \\
\hline $\mathrm{x}=-1$ & 0 & 0 & 0 & 0 \\
\hline $\mathrm{x}=-0,7$ & 0,05381 & 0,10673 & 0,20652 & 0,25194 \\
\hline $\mathrm{x}=-0,4$ & 0,07917 & 0,15772 & 0,31049 & 0,38353 \\
\hline $\mathrm{x}=0$ & 0,08293 & 0,16586 & 0,33151 & 0,41399 \\
\hline $\mathrm{x}=0,4$ & 0,07938 & 0,15939 & 0,32387 & 0,40968 \\
\hline $\mathrm{x}=0,7$ & 0,05412 & 0,10915 & 0,22588 & 0,28978 \\
\hline $\mathrm{x}=1$ & 0 & 0 & 0 & 0
\end{tabular}

Table 2. Approximate solution $\left(u_{\varepsilon}^{N}(x, t), v_{\varepsilon}^{N}(x, t)\right)$ of the approximate data

\begin{tabular}{c|c|c|c|c}
\hline & $\mathrm{t}=0,05$ & $\mathrm{t}=0,1$ & $\mathrm{t}=0,2$ & $\mathrm{t}=0,25$ \\
\hline $\mathrm{x}=-1$ & 0 & 0 & 0 & 0 \\
\hline $\mathrm{x}=-0,7$ & 0,51279 & 0,60612 & $-2,29619$ & $-15,40052$ \\
\hline $\mathrm{x}=-0,4$ & 0,74799 & 1,07263 & 2,21442 & 3,73812 \\
\hline $\mathrm{x}=0$ & 0,08615 & 0,16517 & $-0,14541$ & $-1,77212$ \\
\hline $\mathrm{x}=0,4$ & $-0,57523$ & $-0,69919$ & $-1,47344$ & $-3,22040$ \\
\hline $\mathrm{x}=0,7$ & $-0,38698$ & $-0,29296$ & 3,84245 & 19,74645 \\
\hline $\mathrm{x}=1$ & 0 & 0 & 0 & 0 \\
\hline \hline & $\mathrm{t}=0,05$ & $\mathrm{t}=0,1$ & $\mathrm{t}=0,2$ & $\mathrm{t}=0,25$ \\
\hline $\mathrm{x}=-1$ & 0 & 0 & 0 & 0 \\
\hline $\mathrm{x}=-0,7$ & 0,05382 & 0,10674 & 0,20654 & 0,25196 \\
\hline $\mathrm{x}=-0,4$ & 0,07918 & 0,15773 & 0,31052 & 0,38357 \\
\hline $\mathrm{x}=0$ & 0,08294 & 0,16587 & 0,33154 & 0,41403 \\
\hline $\mathrm{x}=0,4$ & 0,07939 & 0,15941 & 0,32391 & 0,40972 \\
\hline $\mathrm{x}=0,7$ & 0,05412 & 0,10916 & 0,22590 & 0,28981 \\
\hline $\mathrm{x}=1$ & 0 & 0 & 0 & 0 \\
\hline
\end{tabular}

\section{Conclusions}

Results of numerical experiments showed the effectiveness of the proposed approach.

Choosing parameter regularization from the optimality of estimate of the norm of difference between exact and approximate solution give for us possibility to stable calculation. We can see approximate calculation depends from the choosing of correctness set and parameter of regularization.

\section{REFERENCES}

[1] Igor S. Pulkin. Gevrey problem for parabolic equations with changing time direction. Electronic Journal of Differential Equations, Vol. 2006, No.50, pp. 1-9.

[2] Levine H.A. Logarithmic Convexity, First order Differential Inequalities and Some Applications. / Trance. of AMS, v.152, November 1970.

[3] Lavrentev, M.M. Conditional-correct problem for differential equations. Novosibirsk: NGU, 1973. 72 p. (In Russ.)

[4] Tersenov, S.A. Parabolic equations with a varying direction of time. Novosibirsk, 1985. 105 p. (In Russ.)

[5] Fayazov K.S. Ill-posed boundary value problem for a mixedtype equation of the second order. Uzbek Mathematical Journal, 1995. No.2, p. 89-93

[6] Fayazov, K.S., Khazhiev, I.O. Conditional correctness of boundary-value problem for composite fourth-order diferential equation // Izvestiya vuzov, Mathematics, RAS, 2015. No.4, p. 65-74. 